\title{
SPLEEN CONTEMPORAIN ET MAL DE VIVRE EN MILIEU URBAIN DANS (INTERVALLE OUVERT) D'IRINA-ROXANA GEORGESCU
}

\author{
CONTEMPORARY SPLEEN AND MAL DE VIVRE \\ IN IRINA GEORGESCU'S (INTERVALLE OUVERT)
}

\author{
Bouchra BENBELLA' \\ https://doi.org/10.52744/9786062613242.12
}

Résumé : (Intervalle ouvert) est un recueil de poésie doté d'un sens paradoxal et d'une métrique peu usuelle aussi déconcertante qu'originale. Irina-Roxana Georgescu met en mots les maux d'une âme en proie au spleen qui caractérise la mentalité urbaine des temps modernes et contemporains. Présentée sous la forme d'un monologue intérieur, sa poésie est le cri d'un moi féminin cloîtré et mélancolique, mais rêvant d'un voyage susceptible de le soustraire à une existence " de sable », à un réel morose qui broie constamment ses " os blancs et doux».

Mots-clés : Spleen ; Ville ; Crise Existentielle ; Mort ; Poésie ; Voyage.

Abstract: (Intervalle ouvert) is a collection of poetry of a paradoxical significance and an unusual metric both disconcerting and original. IrinaRoxana Georgescu puts into words the evils of a splenetic soul, a distinctive feature of the urban mentality of modern times. Presented in the form of an interior monologue, her poetry is a cry from a cloistered and melancholic feminine self, but dreaming of a journey likely to remove it from a life "of sand", from a gloomy reality that constantly crushes its "white and soft bones".

Keywords: Spleen; City; Existential Crisis; Death; Poetry; Travel

${ }^{1}$ Université Moulay Ismaïl, Meknès, b.benbellao12@gmail.com 
Il est des textes qui hantent le lecteur, le perturbent, l'angoissent et l'intimident. C'est exactement ce que j'ai vécu en lisant à plusieurs reprises (Intervalle ouvert) (Georgescu 2017) d'une jeune poétesse d'origine roumaine : Irina-Roxana Georgescu (n. 1986). A chaque fois que je crois avoir réussi, un tant soit peu, à ouvrir une infime brèche qui me permettrait de me faufiler dans sa conscience et son imaginaire, cette brèche se referme aussitôt que ma lecture du recueil est terminée.

Il me semble en effet que la poésie d'Irina se situe dans un intervalle entre la mémoire et l'oubli, l'identité et la désidentité, le moi et l'autre, le vide et l'amour, le spleen et l'espace urbain, la vie et la mort...

En mathématiques, on parle d'intervalle ouvert quand des nombres sont placés entre deux crochets ouverts, et d'intervalle fermé quand les nombres sont mis entre deux crochets fermés. Georgescu opte pour un Intervalle ouvert qu'elle emprisonne entre deux parenthèses. Un oxymore éloquent à bien des égards, étant donné qu'il annonce dès l'intitulé ce qui sera développé à l'intérieur du recueil : la confusion de l'esprit, le doute, l'illusion d'une ouverture et par conséquent d'une liberté qui prend fin avec la présence des parenthèses, susceptible de générer une amertume immodérée résultant d'une tristesse, d'un vide affectif caractéristique des sociétés modernes. Ce qui justifie la présence de la citation de Tahar Ben Jelloun, mise en épigraphe de l'ouvrage : " Cette soif de posséder et de consommer traduit chez nous un manque immense. Quelque chose d'essentiel nous manque ". D'emblée, la poétesse révèle à son lecteur l'esprit de son recueil, le fil d'Ariane qui lierait tous les thèmes qui y sont abordés. Elle assimile la société de consommation à la réification de l'existence de l'être humain.

On retrouve in medias res, ces vers en écho à cette épigraphe, cette « chose essentielle » qui nous manque :

//là où on s'aime, il ne fait jamais nuit//

(un jour je me souviendrai de tout)

Rejoindre en silence cet amour qui manque à tout amour

(Georgescu 2017, 28)

L'incipit du recueil, première page qui accueille le lecteur, est pratiquement une page blanche ! Que signifie ce blanc, ce silence rompu in extremis par une phrase négative : " Il ne s'agit pas d'un texte d'ouverture et d'accueil / Il n'est pas un préambule (9), niant le recours au prélude dans ce recueil de facture mélancolique ? Sans doute une 
caractéristique de l'écriture poétique de Georgescu qui tente, à travers ce blanc $^{2}$, de rendre visible l'invisible, nourrissant pour ainsi dire une polysémie idoine, une confusion propre à sa poésie. Cette confusion concrétise un manque, " un vide comme espace tragique que n'anime aucune trace, ou l'intervalle, place perdue entre deux figures » (SimonOikawa 2010), rattachant l'incipit à l'épigraphe. Aussi, joue-t-elle de la spatialité de ses vers et de leur disposition sur la page blanche pour rendre visible sa mélancolie causée par un manque, une séparation avec un ami anonyme, son interlocuteur, qui ne réagit jamais à ses interpellations. (Où es-tu ?) (Georgescu 2017, 9), (Où es-tu vraiment ?) (Georgescu 2017, 13), (est-ce que tu es encore là ?) (Georgescu 2017, 15). Ce qui suggère un monologue intérieur qui participe de l'expression des pensés les plus intimes de la poétesse. Des pensées qui traversent son âme dans un flux ininterrompu au fur et à mesure qu'elles surgissent, se dérobant à tout enchaînement logique, à toute préoccupation raisonnante, n'obéissant qu'à la spontanéité dans laquelle elles naissent et à une structure phrastique concise et directe : " Le monologue intérieur est, dans l'ordre de la poésie, le discours sans auditeur et non prononcé, par lequel un personnage exprime sa pensée la plus intime, la plus proche de l'inconscient, antérieurement à toute organisation logique, c'est-à-dire en son état naissant, par le moyen de phrases directes réduites au minimum syntaxial, de façon à donner l'impression 'tout-venant' » (Dujardin 2017). Son monologue intérieur, Georgescu l'assimile à une confession salvatrice, cathartique et purificatoire :

La confession fait bien / / la confession est un cri d'identité corrodant quelque chose en nous.

(Georgescu 2017, 23)

Dans ce cri de la pensée intime, de l'inconscient, la poétesse ressent l'obligation d'entamer un long voyage avec son destinataire anonyme à qui elle s'adresse tout au long de ce poème dans un échange unilatéral. Â la lumière de la philosophie du dialogue qui insiste sur l'idée de la réciprocité, sa tentative est vouée à l'échec face au silence du partenaire. Elle s'efforce dans ce poème en prose monodique d'établir un dialogue avec son destinataire (son bien-aimé ?) séparé par la distance :

\footnotetext{
2 «Que l'on tende à l'interpréter plutôt d'une manière ou d'une autre, le "blanc" est toujours simultanément vide et plein à la fois, signe avant-coureur d'un invisible à ce point inconcevable et dense qu'il peut être perçu comme une absence, mais aussi don de lumière total et immédiat, spectacle d'emblée absolu de tous les possibles réalisables. Telle est l'énigme ». Anne-Marie Christin, Poétique du blanc : vide et intervalle dans la civilisation de l'alphabet, Peeters-Vrin, 2000. p.8.
} 
(Où es-tu ?)

Je viens de te revoir.

Je viens de te dire que j'ai vécu tout ce temps avec l'image de

Nos retrouvailles

Ce temps est passé. (Georgescu 2017, 9-10)

« Je t'ai cherché, et je ne t’ai point trouvé // je t'ai appelé, et tu ne m'as point répondu. » (Georgescu 2017, 24)

Ce voyage qu'elle veut entreprendre avec son partenaire est en fait avant tout une fuite devant la mélancolie dont elle souffre en permanence : " Partir vers l'inconnu est une motivation essentielle dans le texte d'Irina Georgescu, et qui consiste à agrémenter le départ comme étant une possibilité de renouvellement ailleurs pour pouvoir mieux camper son identité dans le milieu désiré » (Benkirane 2018). Aussi, un mouvement très proustien s'opère-t-il dans ce recueil. Mouvement du doute généralisé : " On doit laisser les certitudes derrière nous » (Georgescu 2017, 10). Mouvement de la crise angoissante suscitée par la dissolution de l'« identité » et des certitudes, qu'elle nomme « certitudes de sable » (Georgescu 2017, 51) et qui s'attachent à elle comme une deuxième peau (Georgescu 2017, 45) :

Cette lecture nous renvoie à nos propres images intérieures (en permanence, la question gravée au-dessous de ma peau / / qui suis-je ? / / comme un tambour qui éclate au néant) Ton confidentiel, amitié, magie, poésie, amour un véritable morceau de vie / / \& de littérature

(Georgescu 2017, 24)

Tout porte à croire qu'il s'agit d'une remise en question identitaire et de la manifestation d'une crise existentielle liée à la perte des repères. La douleur émotionnelle omniprésente dans le poème mène à une rupture - rupture avec son réel amer et mélancolique, dont elle déverse l'encre dans cette écriture poétique, épanchant le mal et laissant couler la plainte. L'affirmation de l'identité personnelle du « je » féminin s'avère ambiguë dans la mesure où ce « je » lyrique se concentre essentiellement sur les aspects négatifs de la vie, à un point tel que l'inaptitude à s'accrocher à une représentation nettement positive de soi ne fait que le pousser plus loin dans sa mélancolie :

À contrecœur la tristesse que je porte en moi comme une seconde peau c'est un torrent ; nier ce sentiment, étouffant ce mirage du destin chanceux, redécouvrir cette passion ouvragière, inconstante et diffuse d'où ce flux de paroles, d'où cette attente lumineuse de l'aube

(Georgescu 2017, 22) 
Cette mélancolie, les théoriciens tels que Simmel et Kracauer (Füzesséry et Simay 2008) la reconnaissent comme un trait distinctif de la mentalité urbaine des temps modernes. Elle est diagnostiquée comme source majeure de l'aporie de la vie de l'homme contemporain. Sur ce point, Irina Georgescu se rapproche de Baudelaire qui considérait le « moderne » comme le spleen de l'espace urbain : lieu d'une désintégration, d'une vaporisation du moi :

Il y a chez Baudelaire un substrat social, moderne, qui joue un rôle déterminant dans l'idylle funèbre de la ville. Le moderne est un accent capital de sa poésie. En l’identifiant au spleen, Baudelaire fait éclater l'idéal. ${ }^{3}$

Toujours est-il que la poésie demeure une forme esthétique d'une ascension morale que la poétesse oppose à la réalité spleenétique, lasse et anxieuse de sa vie habituelle dans sa ville ou en proie à la solitude dans sa chambre :

Au gouffre, mon cher ami, au gouffre, je te dis / / la poésie nous envahit les sens, les rêves, les voies, la poésie devient ombre et puis souffle vital.

(Georgescu 2017, 37)

Il difficile de détecter la raison de l'utilisation de l'écriture en italique de ces trois vers ; cela évoquerait peut-être une didascalie, cette instruction que l'auteur donnerait aux acteurs de son théâtre formant pour ainsi dire un " hors-texte ». Le même procédé est réemployé à plusieurs reprises dans le recueil, rappelant à bien des égards le théâtre comme genre littéraire :

Dimanche. Dans une rue calme, une femme vêtue d'un élégant habit noir semble quitter la scène. Derrière elle, un cocher attend avec calme dans la voiture de son maître.

(Georgescu 2017, 27)

Cette séquence prosaïque est similaire à une didascalie initiale qui chapeaute une scène où la poétesse-dramaturge fait jouer des figures picturales des tableaux de Botero et de Niki de Saint Phalle. Des figures féminines qui sont une allusion aux formes rondes et généreuses du corps, contrastant avec la géométrie rectiligne et monotone de la ville. Il est aussi à remarquer que cette didascalie rappelle vaguement mais ad libitum, la rencontre fortuite du poète dans «Une passante » de Baudelaire, peintre des « Tableaux parisiens » de la grande ville moderne laide et désespérante :

3 « Paris, Capitale du XIX ${ }^{\mathrm{e}}$ siècle », in Walter Benjamin, Euvres IV, Paris, Folio, coll., Essais, 2000, p.47. 
La rue assourdissante autour de moi hurlait.

Longue, mince, en grand deuil, douleur majestueuse,

Une femme passa, d'une main fastueuse

Soulevant, balançant le feston et l'ourlet.

Seulement, la ville chez Georgescu n'est pas assourdissante, elle n'est pas perçue à travers la foule, que Walter Benjamin assimile à un voile constitué par la masse, en tant que nouveau mode d'être de la multitude (Déotte 2008). L'espace urbain dans ce recueil est perçu comme calme, monotone et mélancolique :

Une rue de Moscou, de Bologne ou de Bucarest a la même

Mélancolie muette, le même silence dans la plus noircie nuit de

Toutes les nuits, quand

La violence existe sans provenir du réel quand

La contrainte même se banalise

Une rue de Bucarest reste l'écho de ce dimanche banalisé auquel

Je me colle

(Georgescu 2017, 20)

La rue calme autour de la poétesse hurle, une placidité très allusive ne fait que la renvoyer à elle-même, à sa mélancolie, à ce sentiment de vide qui ronge ses « os blancs et doux » (26).

Georgescu dépeint ses activités monotones pendant les jours de la semaine, ponctuées par le travail. Une monotonie accentuée par la morosité de la saison hivernale :

C'est samedi. Le jour est passé comme s'il n'avait jamais existé

(on travaille même les samedis et les dimanches

on travaille bien

on travaille inutilement)

Ou encore :

(Georgescu 2017, 19)

Dans cette matinée grisâtre de mardi, je me dépêche vers le boulot

/ / blocage / /

je suis encore ensommeillée, mais le froid me réveille

Tous ces gens autour de moi,

/ déjà fatigués /

se dépêchent eux aussi vers le boulot

Chacun avec son propre amour frissonné, avec sa propre

serviette noire et quelques papiers qui attestent leur âge et leur

statut social

La nature humaine se technicise dans sa férocité aimable

La nature humaine devient la nature des objets

Le sommeil aux couleurs de l'aube m'accompagne

Combien de temps a-t-on attendu pour arriver ici ?

(Georgescu 2017, 23) 
Tout en cultivant dans son recueil la poésie du quotidien, la poétesse dépeint la tragédie de l'homme-objet dans la société contemporaine. La vision du Moi poétique dans ce texte est terrestre et réaliste et non métaphysique ou onirique. Il y dénonce l'exploitation de l'homme, sa réification, l'extinction de son humanité à cause de cette religion de la modernité technique qui relève de la plus énormes des impostures, ce " fanal obscur », " cette idée grotesque, qui a fleuri sur le terrain pourri de la fatuité moderne » (Baudelaire 1868). Le progrès purement matériel, roue au double engrenage qui sans cesse fait marcher quelque chose en écrasant quelqu'un, pour reprendre une sentence de Victor Hugo, est en réalité la preuve de la décadence dans laquelle sombre l'humanité.

Les gens oublient qu'ils vivent

À l'ombre de leur vie,

dans un paradoxe qui frissonne et fouette et miaule

de temps en temps comme une poupée mécanique

(Georgescu 2017, 43)

La stigmatisation de la société moderne dans ce poème, montrant l'homme et la femme esclaves de leur travail, rappelle à bien des égards ces vers de Jacques Prévert :

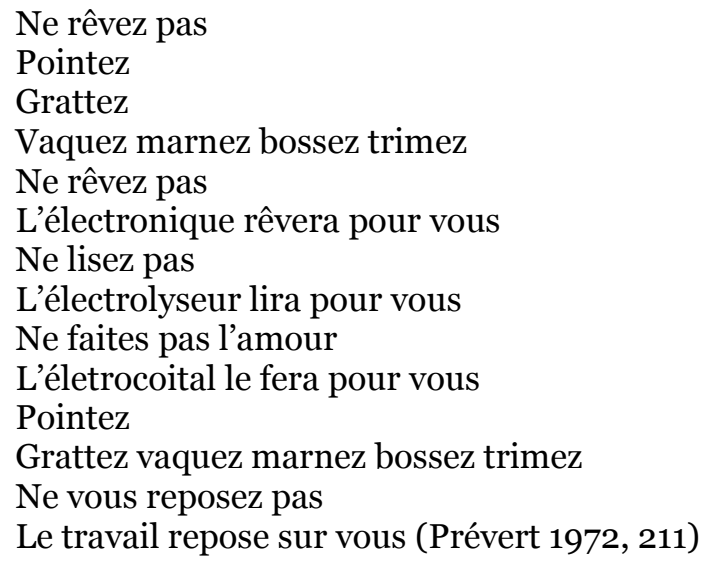

L'humanité est ainsi plongée dans ce que Hegel nomme « le monde du quotidien et de sa prose ». L'aliénation inconditionnelle au travail façonne le corps des hommes en les éloignant de l'idéal, c'est-à-dire en réifiant leur individualité, en supprimant la liberté sous le masque du caractère acquis, qui est caricature 4 .

4 http://www.jdarriulat.net/Auteurs/Hegel/HegelIdeeBeau.html 
En somme, ce recueil pourrait se lire comme un intervalle où la vie compose avec le silence la symphonie de la solitude (Benkirane 2018). Le cri d'un moi féminin cloîtré et mélancolique, mais rêvant d'un départ susceptible de le soustraire à une existence " de sable ", à un réel désappointant qui broie constamment ses « os blancs et doux ».

La métrique peu usuelle de la poésie d'Irina Georgescu révèle sa musique intérieure au rythme des barres obliques qui la délinéent, exprimant à la fois la meurtrissure interne, l'union, la (con)fusion ou encore l'étouffement. Un souffle coupé, un flux scandé marquant ce passage, cet intervalle entre mort et vie.

\section{Bibliographie}

Baudelaire, Charles. (1868). "Exposition universelle, 1855. Beaux-arts » dans Euvres complètes de Charles Baudelaire, vol. II (p. 211-244). Paris : Michel Lévy frères.

Benjamin, Walter. (2000). CEuvres IV. Paris : Folio.

Benkirane, Kamal. (2018). « Le labyrinthe comme poème dans « (Intervalle ouvert) » de Irina-Roxana Georgescu, Revue en ligne Possibles, 17 avril 2018. http://redtac.org/possibles/2018/o4/17/ le-labyrinthe-comme-poeme-dansintervalle-ouvert-de-irina-roxana-georgescu/

Christin, Anne-Marie. (2000). Poétique du blanc : vide et intervalle dans la civilisation de l'alphabet. Paris : Peeters-Vrin.

Deotte, Jean-Louis. (2008). « La ville appareillée : Arendt, W. Benjamin et Baudelaire », Appareil [En ligne], Numéro spécial, 2008, consulté le 19 avril 2020. URL : http://journals.openedition.org/appareil/449 ; DOI : https://doi.org/ 10.4000/appareil.449

Dujardin, Edouard. (2017). "ANNEXE », Modèles linguistiques [En ligne], 76 : document 9, consulté le 05 avril 2020. URL : http://journals.openedition. $\mathrm{org} / \mathrm{ml} / 5328 ;$ DOI : https://doi.org/10.400o/ml.5328

Füzesséry, Stéphane et Philippe Simay, (éd.). (2008). Le choc des métropoles. Simmel, Kracauer, Benjamin et la Grossstadt. Paris : Éd. de l'Eclat.

Georgescu, Irina Roxana. (2017). «Intervalle ouvert ». Paris : L'Harmattan.

Prévert, Jacques. (1972). "L'ordinateur », Choses et autres. Paris : Gallimard.

Simon-Oikawa, Marianne. (2010). «Une poétique du blanc », Acta fabula, 11(4), Notes de lecture, Avril 2010, URL : http://www.fabula.org/revue/document 5613.php, page consultée le 16 avril 2020.

http://www.jdarriulat.net/Auteurs/Hegel/HegelIdeeBeau.html 